\title{
A NOTE ON WEINSTEIN TRANSFORM ON PRODUCTS OF CENTRAL MORREY SPACES
}

\author{
MARTHA GUZMÁN-PARTIDA \\ Date of Receiving : $\quad 09.01 .2020$ \\ Date of Revision : 06.04 .2020 \\ Date of Acceptance : 08.05 .2020
}

\begin{abstract}
We prove boundedness of convolution operators given by product domain versions of the Weinstein transform acting on products of central weighted Morrey spaces.
\end{abstract}

\section{Introduction}

The aim of this note is to obtain boundedness results on some function spaces of convolution operators induced by a product version of the kernel $\mathscr{K}_{\alpha}$, defined by

$$
\mathscr{K}_{\alpha}(y, s):=\frac{\Gamma((\alpha+n+1) / 2)}{\Gamma((\alpha+1) / 2) \pi^{n / 2}} \frac{s^{\alpha+1}}{\left(|y|^{2}+s^{2}\right)^{(\alpha+n+1) / 2}}
$$

where $\alpha>-1$ and $(y, s) \in \mathbb{R}_{+}^{n+1}$.

This kernel is related to the elliptic partial differential equation

$$
D_{\alpha} u:=s^{-\alpha}\left(\frac{\partial^{2} u}{\partial y_{1}^{2}}+\ldots+\frac{\partial^{2} u}{\partial y_{n}^{2}}+\frac{\partial^{2} u}{\partial s^{2}}-\frac{\alpha}{s} \frac{\partial u}{\partial s}\right)=0
$$

with $\alpha>-1$. Solutions to (1.2) are called generalized axially symmetric potentials (cf. [13]). Notice that, for $\alpha=0$ we recover the Laplace equation.

Several authors have approached the study of boundary value problems related to this kernel, as well as modified versions of it (see, for example, [14],[2],[3], [6]).

As it happens in the case of the Poisson kernel for the upper half-space, it can also be proved that: (i) $\left\|\mathscr{K}_{\alpha, s}\right\|_{L^{1}}=1$, where $\mathscr{K}_{\alpha, s}(y):=\mathscr{K}_{\alpha}(y, s)$, (ii) $\mathscr{K}_{\alpha, s} \rightarrow \delta_{0}$ in $\mathscr{S}^{\prime}$ as $s \rightarrow 0^{+}$, and (iii) $\mathscr{K}_{\alpha}$ is a solution to the equation (1.2) in $\mathbb{R}_{+}^{n+1}$ (see [14]). In that article, generalizing techniques developed in [3] and [2], the author characterizes a

2010 Mathematics Subject Classification. 42B35, 44A35.

Key words and phrases. Weinstein operator; product central Morrey spaces.

Communicated by: Nikhil Khanna

${ }^{\dagger}$ Corresponding author. 